\title{
ANNIHILATORS IN SEMIPRIME RIGHT GOLDIE RINGS
}

\author{
by C. R. HAJARNAVIS
}

(Received 20th March 1990)

(i) Let $R$ be a semiprime right Goldie ring with $\operatorname{dim} R=n$. Then a maximal chain of right annihilators in $R$ has exactly $n$ terms. (ii) A semiprime locally right Goldie ring with ACC and DCC on right annihilators is a right Goldie ring.

1980 Mathematics subject classification (1985 Revision): 16A34.

\section{Introduction}

The aim of this note is to answer two questions raised by Brown and Wehrfritz in [1].

Let $R$ be a semiprime right Goldie ring of uniform dimension $n$. Since the right singular ideal of $R$ is zero, the right annihilators are complements and so any ascending (or descending) chain of right annihilators in $R$ has at most $n$ terms. While studying certain crossed products, Brown and Wehrfritz have wondered if a maximal such chain necessarily has $n$ terms. This is easy to see if the Goldie chain conditions are assumed to be two-sided. In this case $R$ has a two-sided quotient ring $Q$ of dimension $n$. The extension of a chain of right annihilators in $R$ can be refined to a chain of length $n$ of right ideals in $Q$. Intersecting back to $R$ we get a chain of length $n$ in which every term is a right annihilator of a single element of $R$; this last property following from the fact that elements of $Q$ are expressible as left fractions.

We demonstrate here that although the above strategy fails under one-sided assumptions, further analysis of right complements in $R$ shows the required result to be true (Theorem 3.4). We also show that if we make the extra assumption that left regular elements of $R$ are right regular then complement right ideals are right annihilators (Theorem 3.6). Thus in this case contraction to $R$ of a composition series in $Q$ does give a chain of right annihilators.

In answer to the second question from [1] we show that a semiprime locally right Goldie ring with ACC and DCC on right annihilators is itself a right Goldie ring (Theorem 4.1).

It is a pleasure to thank Alfred Goldie and the referee for their helpful suggestions.

\section{Preliminaries and definitions}

Let $S$ be a non-empty subset of a ring $R$. Then $r(S)=\{x \in R \mid S x=0\}$ is called the right 
annihilator of $S$. A right ideal is said to be a right annihilator if it is the right annihilator of some subset of $R$. The left annihilator of $S$ denoted $l(S)$ is defined analogously. We shall abbreviate $r(l(S))$ to $r l(S)$. An element $c \in R$ is called right regular if $r(c)=0$ and left regular if $l(c)=0$.

A submodule $C$ of a right module $M$ is said to be a complement if $C$ has no essential extension in $M$.

The term dimension will refer to the uniform (Goldie) dimension of the module $M$ and will be denoted by $\operatorname{dim} M$.

The maximal complements in $M$ are precisely the complements of dimension one less than $\operatorname{dim} M$.

The abbreviations ACC and DCC will stand for the ascending chain condition and the descending chain condition respectively.

A right ideal $I$ of a ring $R$ is said to be closed if $x c \in I$ with $x \in R$ and some $c$ regular in $R$ implies that $x \in I$.

When $R$ is a semiprime right Goldie ring with a right quotient ring $Q$ it is standard to show that right annihilators are complements and $I$ is a complement $\Leftrightarrow I$ is closed $\Leftrightarrow$ $I=J \cap R$ for some right ideal $J$ of $Q$.

We refer the reader to [2] and [3] for further background information. The methods followed are those of [3]. Since these notes are now not readily available, we have included some detail here and given alternative references where possible.

\section{Complements and annihilators}

We begin by stating some preliminary facts.

Lemma 3.1 [4, Theorem 1.5]. Let $C$ be a complement in a finite-dimensional faithful module $M$. Then $C$ is expressible as an intersection of maximal complements. Such an expression when irredundant has exactly $\operatorname{dim} M-\operatorname{dim} C$ terms.

Lemma 3.2 [4, Theorem 3.7]. Let $Y$ be a subset of a semiprime right Goldie ring $R$. Let $\operatorname{dim} R_{R}=n$ and $\operatorname{dim} r(Y)=k$. Then there exist uniform right ideals $U_{1}, U_{2}, \ldots, U_{n-k}$ and elements $u_{i} \in U_{i}$ such that $r(Y)=r\left(u_{1}\right) \cap \cdots \cap r\left(u_{n-k}\right)$.

Lemma 3.3. Let $U$ be a uniform right ideal of a semiprime right Goldie ring $R$.

(i) If $r(S)$ is a right annihilator and $U \cap r(S) \neq 0$ then $U \subseteq r(S)$.

(ii) For every $a \in R$ we have $\operatorname{dim} a R+\operatorname{dim} r(a)=\operatorname{dim} R$.

(iii) If $0 \neq u \in U$ then $r(u)$ is a maximal complement.

Proof. (i) Note that $U \cap r(S)$ is essential in $U$. The result follows using [2, Lemma 1.1] and the fact that the right singular ideal of $R$ is zero.

(ii) Let $\operatorname{dim} R_{R}=n$ and $\operatorname{dim} r(a)=k$. Let $U_{1} \oplus \cdots \oplus U_{k} \subseteq r(a)$ be an essential direct sum of uniform right ideals of $R$. Extend this to a direct sum of uniform right ideals 
$U_{1} \oplus \cdots \oplus U_{k} \oplus U_{k+1} \oplus \cdots \oplus U_{n}$ such that $r(a) \cap\left(U_{k+1} \oplus \cdots \oplus U_{n}\right)=0$ and $U_{1} \oplus \cdots \oplus U_{n}$ is essential in $R$. It is easy to see that $a U_{k+1}+\cdots+a U_{n}$ is a direct sum of non-zero submodules. Since $a U_{i} \neq 0$ for $i>k$, applying (i) to the kernel of the canonical map from $U_{i}$ to $a U_{i}$ shows that $U_{i} \cong a U_{i}$. Hence each $a U_{i}$ is uniform. Now let $X \subseteq a R$ be a non-zero right ideal. Let $Y=\{y \in R \mid a y \in X\}$. Then $Y$ is a right ideal of $R$ and $a Y=X$. Hence $Y \neq 0$ and so $Y \cap\left(U_{1} \oplus \cdots \oplus U_{n}\right) \neq 0$. It is easy to deduce from this that $X \cap\left(a U_{k+1} \oplus \cdots \oplus a U_{n}\right) \neq 0$. Thus $a U_{k+1} \oplus \cdots \oplus a U_{n}$ is an essential direct sum of uniform right ideals in $a R$ and so $\operatorname{dim} a R=n-k$.

(iii) By the above $\operatorname{dim} u R+\operatorname{dim} r(u)=\operatorname{dim} R$. Since $u R$ is uniform we have $\operatorname{dim} r(u)=\operatorname{dim} R-1$. Thus $r(u)$ is a maximal complement.

As noted in the introduction, the length of a chain of right annihilators in a semiprime right Goldie ring is bounded by the dimension of the ring. Thus any such chain can be refined to a maximal one.

Theorem 3.4. Let $R$ be a semiprime right Goldie ring of dimension $n$. Then any maximal chain of right annihilators in $R$ has exactly $n$ terms.

Proof. Let $r(X) \subsetneq r(Y)$ be two right annihilators chosen so that no right annihilator lies strictly between $r(X)$ and $r(Y)$. Let $\operatorname{dim} r(X)=s$ and $\operatorname{dim} r(Y)=k$. In order to prove the theorem it suffices to show that $s=k-1$. Since $r(X)$ is a complement $s<k$. So there exists a uniform right ideal $U \subseteq r(Y)$ such that $U \cap r(X)=0$. We shall next show that there exists $u \in U$ with the properties that $r(u) \supseteq r(X)$ and $U \cap r(u)=0$. We may without loss of generality assume that $X$ is a left ideal. Clearly for any $v \in X \cap U$ we have $r(v) \supseteq r(X)$. Suppose now that $r(v) \cap U \neq 0$. Then $U \subseteq r(v)$ by Lemma 3.3. Thus if $r(v) \cap U \neq 0$ for all $v \in X \cap U$ then we have $(U \cap X) U=0$. This gives $U X U=0$ and so $(X U)^{2}=0$. But then $X U=0$ and $U \subseteq r(X)$ which is a contradiction. It follows that we can choose $u \in U$ such that $r(u) \supseteq r(X)$ and $r(u) \cap U=0$.

Now we have $r(X) \subseteq r(Y) \cap r(u) \subseteq r(Y)$. By assumption this implies that either $r(X)=r(Y) \cap r(u)$ or $r(Y) \cap r(u)=r(Y)$. This latter possibility gives $r(Y) \subseteq r(u)$. Since $U \subseteq r(Y)$ it follows that $U \subseteq U \cap r(u)=0$ which is a contradiction. Thus we have $r(X)=$ $r(Y) \cap r(u)$. Expressing $r(Y)$ as in Lemma 3.2 we obtain $r(X)=r\left(u_{1}\right) \cap \cdots \cap r\left(u_{n-k}\right) \cap r(u)$. By Lemma $3.3 r(u)$ and the $r\left(u_{i}\right)$ are maximal complements. By Lemma 3.1 we must have $n-s \leqq n-k+1$ and so $s \geqq k-1$. Thus $s=k-1$.

In [3] Goldie has shown that in a semiprime left and right Goldie ring the complement right ideals are right annihilators. Theorem 3.4 follows easily from this when chain conditions are assumed on both sides. The example in [4, p. 220] shows that a maximal right complement need not be a right annihilator under one-sided assumptions. This shows in particular that a chain of right annihilators in a semiprime right Goldie ring cannot be refined to a maximal one simply by inserting complements. The starting point of the above example is the existence in the ring of a left regular element which is not right regular. It is of some interest to note that this is, in fact, the only obstruction. 
Lemma 3.5. Let $R$ be a semiprime ring with ACC and DCC on right annihilators. Let $I$ be a right ideal of $R$ such that $l(I)=0$. Then I contains a left regular element of $R$.

Proof. $R$ also has ACC on left annihilators so by [5, Theorem 1] nil subrings of $R$ are nilpotent. Since $l(I)=0, I$ is not nilpotent and so it is not nil. Choose $x_{1} \in I$ such that $l\left(x_{1}\right)$ is maximal among the left annihilators of non-nilpotent elements of $I$. Then $l\left(x_{1}^{2}\right)=l\left(x_{1}\right)$. If $l\left(x_{1}\right)=0$ then $x_{1}$ is the required element. Suppose that $l\left(x_{1}\right) \neq 0$. Then $l\left(x_{1}\right) \cap I$ is not nilpotent since $\left[l\left(x_{1}\right) \cap I\right]^{k}=0 \Rightarrow\left[I l\left(x_{1}\right)\right]^{k}=0 \Rightarrow\left[l\left(x_{1}\right) I\right]^{k+1}=0 \Rightarrow l\left(x_{1}\right) I=$ $0 \Rightarrow l\left(x_{1}\right)=0$ which is a contradiction. Hence $l\left(x_{1}\right) \cap l$ is not nil.

Choose $x_{2} \in l\left(x_{1}\right) \cap I$ such that $l\left(x_{2}\right)$ is maximal among the left annihilators of non-nilpotent elements of $l\left(x_{1}\right) \cap I$. Then $l\left(x_{1}+x_{2}\right)=l\left(x_{1}\right) \cap l\left(x_{2}\right)$ for $t\left(x_{1}+x_{2}\right)=0$, $t \in R \Rightarrow t x_{1}=-t x_{2} \Rightarrow t x_{1}^{2}=-t x_{2} x_{1}=0 \Rightarrow t \in l\left(x_{1}^{2}\right)=l\left(x_{1}\right) \Rightarrow t x_{1}=t x_{2}=0$. Also $l\left(x_{1}\right) \supsetneq l\left(x_{1}+x_{2}\right)$ for $x_{2} x_{1}=0$ but $x_{2}^{2} \neq 0$.

If $l\left(x_{1}+x_{2}\right) \neq 0$ we proceed as before. By DCC on left annihilators this process must stop to give $x=x_{1}+x_{2}+\cdots+x_{n} \in I$ such that $l(x)=0$.

Theorem 3.6. Let $R$ be a semiprime right Goldie ring. Then the following statements are equivalent.

(i) Every complement right ideal is a right annihilator.

(ii) $I$ is essential in $r(I)$ for every right ideal $I$.

(iii) Every left regular element is right regular.

Proof. By [2, Corollary 1.15] $R$ also has DCC on right annihilators.

(iii) $\Rightarrow($ i $)$. By Lemma 3.1 it is enough to show that a maximal right complement of $R$ is a right annihilator. Let $I$ be a maximal right complement. Then $I$ is not essential. So by $[2$, Lemma 1.11] $I$ does not contain a right regular element. Hence $I$ does not contain a left regular element and so by Lemma 3.5 we must have $l(I) \neq 0$. Hence $r l(I) \neq R$. Now $r l(I)$ is a complement and $r l(I) \supseteq I$. Since $I$ is a maximal complement it follows that $r l(I)=I$.

$(i) \Rightarrow(i i)$. Let $I$ be a right ideal. Then $I$ is essential in a complement right ideal $K$. Now we have $I \subseteq r l(I) \subseteq r l(K)=K$. Thus $I$ is essential in $r l(I)$.

(ii) $\Rightarrow($ iii). Let $c \in R$ be a left regular. Then $c R$ is essential in $r l(c R)=R$. It follows by [2, Lemma 1.17] that $c$ is right regular.

\section{Locally Goldie rings}

The following definition from [1] conforms to the standard usage in group theory.

Definition. A ring $R$ is said to be a locally right Goldie ring if every finite subset of $R$ lies in a right Goldie subring of $R$. 
Propositions 2, $2^{\prime}$ and 3 of [1] are special cases of our next theorem.

Theorem 4.1. Let $R$ be a semiprime locally right Goldie ring with $\mathrm{ACC}$ and $\mathrm{DCC}$ on right annihilators. Then $R$ is a right Goldie ring.

Proof. We shall first show that $R$ has a right quotient ring. Let $a, c \in R$ with $c$ regular in $R$. Let $I$ be a non-zero right ideal of $R$. Choose $0 \neq b \in I$. Now by assumption there exists a right finite-dimensional subring $S$ of $R$ which contains both $b$ and $c$. Then by [2, Lemma 1.11], $c S \cap B \neq 0$ where $B$ is the right ideal generated by $b$ in $S$. Since $B \subseteq I$, it follows that $c R \cap I \neq 0$. Thus $c R$ is essential in $R$. Hence by [2, Lemma 1.1] so is the right ideal $F=\{x \in R \mid a x \in c R\}$. By [6, Theorem or 2, Theorem 1.19], every essential right ideal of $R$ contains a regular element. Hence $F$ contains a regular element $c_{1}$ say. Thus $a c_{1}=c a_{1}$ for some $a_{1} \in R$ and $R$ has a right quoteint ring.

Let $E$ be an essential right ideal of $Q$ the right quotient ring of $R$. Then $E \cap R$ is essential in $R$. As above $E \cap R$ contains a regular element of $R$. Hence $E=Q$ and $Q$ is a semisimple Artinian ring. It is now standard to show that $R$ must be a right Goldie ring.

Added in proof. Theorem 3.4 can also be proved without reference to Lemma 3.2

\section{REFERENCES}

1. K. A. Brown and B. A. F. Wehrfritz, Goldie criteria for some semiprime rings, J. Glasgow Math. Soc., to appear.

2. A. W. Chatters and C. R. Hajarnavis, Rings with chain conditions (Research notes in mathematics, Pitman 1980).

3. A. W. Goldie, Rings with maximum condition (Lectures given at Yale University, Yale, 1961).

4. A. W. Goldie, Semi-prime rings with maximum condition, Proc. London Math. Soc. (3) 10 (1960), 201-220.

5. I. Herstein and L. W. Small, Nil rings satisfying certain chain conditions, Canad. J. Math. 16 (1964), 771-776.

6. R. E. Johnson and L. S. Levy, Regular elements in semiprime rings, Proc. Amer. Math. Soc. 19 (1968), 961-963.

Mathematics Institute

UNIVERSITY OF WARWICK

Coventry CV4 7AL

UNITED KINGDOM 